\title{
Análisis microestructural de cinco sistemas de implantes de titanio comercialmente puro
}

\author{
Microstructural analysis of five commercially pure titanium implant \\ systems
}

\author{
OLATE $S *$ \\ DUQUE DE MIRANDA CHAVES NETTO H** \\ DE ALBERGARIA-BARBOSA JR ***
}

\begin{abstract}
Olate S, Duque de Miranda Chaves Netto H, de Albergaria-Barbosa JR. Análisis microestructural de cinco sistemas de implantes de titanio comercialmente puro. Av Periodon Implantol. 2010; 22, 1:37-43.
\end{abstract}

\begin{abstract}
RESUMEN
Hoy en día existe un gran número de empresas fabricantes de implantes así como diferentes tipos de implantes, lo que ha llevado a la diversificación en las técnicas de producción, no siempre asociados a una mejor calidad. El objetivo de este estudio fue identificar y tipificar cinco implantes de titanio comercialmente puro. Se utilizó un estudio descriptivo, doble ciego, para estudiar 15 muestras de implantes de 5 empresas comerciales. Cada implante fue removido de su transporte manteniendo sus características originales. Cada grupo fue de cinco implantes, nombrados con las letras A, B, C,D y E. el análisis incluyó metalografia, microscopia electrónica con sistema de microanálisis (SEM-EDS) y finalmente, un analizador de imágenes. Implamed® y Master Screw ${ }^{\circledR}$ mostraron granos de menor tamaño. En microfotografías, todos exhibieron picos de titanio, carbono y oxígeno. Implamed $®$ mostró también acero, sodio, magnesio, silicona, potasio e inclusiones de calcio. Todas las muestras mostraron buena finalización. Las variaciones observadas no serían suficientes para interferir en el proceso de oseointegración, no obstante las inclusiones de metal deben ser estudiadas detalladamente en sus efectos a largo plazo.
\end{abstract}

PALABRAS CLAVE: Implantes dentales, análisis microestructural, titanio.

\section{SUIMIMARY}

Nowadays there is a great number of manufacturers and types of implants which has led to several techniques in their production, not always associated with better quality. The aim of this research was identify and typify five commercially pure titanium implant systems. A double-blind, descriptive trial was used with the selection of 15 samples from 5 different manufacturers. Each implant was removed from its package keeping its original characteristics. Five implant groups of 3 elements each were formed and named A, B, C, D, and E. The analyses included metallography, scanning electron microscopy with a microanalysis system (SEM-EDS) and, finally, an image analyzer. Implamed $₫$ and Master Screw $®$ showed smaller grain sizes. In micrographies, they all showed peaks of titanium, carbon, and oxygen. Implamed $囚$ showed iron, sodium, magnesium, silicon, potassium, and calcium inclusions. All samples showed good finishing. Variations observed would not be enough to interfere with osseointegration but metal inclusions should be studied in greater detail in order to assess its effects in the long run.

KEY WORDS: Dental implants, microstructural analyses, titanium.

Fecha de recepción: 9 de enero de 2009.

Fecha de aceptación: 14 de enero de 2009.

* Profesor, Departamento de Odontología Integral. Facultad de Medicina. Universidad de La Frontera, Chile y Estudiante de Doctorado División de Cirugía Oral y Maxilofacial. Facultad de Odontología de Piracicaba. Universidad Estadual de Campinas. Brasil.

** Estudiante de Doctorado. División de Cirugía Oral y Maxilofacial. Facultad de Odontología de Piracicaba. Universidad Estadual de Campinas. Brasil.

*** Profesor Titular. División de Cirugía Oral y Maxilofacial. Facultad de Odontología de Piracicaba. Universidad Estadual de Campinas. Brasil. 


\section{INTRODUCCIÓN}

La oseointegración depende de múltiples factores. Se ha señalado que después de la técnica quirúrgica, las condiciones del huésped y el tipo de carga en el implante, tendría un papel fundamental en el proceso de oseointegración el tipo de material, el diseño del mismo y las condiciones de superficie (1). La relación entre superficie del implante y el desarrollo de oseointegración es tan relevante que la microtopografía, el sustrato topográfico y la capa de óxido de titanio contribuirían a la regulación de la diferenciación de los osteoblastos y al modelo de respuesta ósea $(2,3)$, así como también a la incorporación de diferentes microorganismos del ecosistema oral cuando se produce su contaminación (4).

Los procesos de limpieza y esterilización pueden producir cambios en los padrones de expresión celular asociada a oseointegración; Ameen et al. (5), en un estudio de espectroscopia fotoelectrónica por rayos $\mathrm{x}$ evaluaron dos superficies de implantes de titanio comercialmente puro, concluyendo que en los discos de superficie rugosa se encontró una considerable contaminación. Edward y Gold (6) evaluaron la superficie de tres sistemas de implantes de titanio comercialmente puro, observando diferencias considerables en su composición, así como también en los grados de contaminación, los cuales dependían principalmente del fabricante. Estos hallazgos están relacionados con el hecho de que diferentes tratamientos sobre materiales homogéneos proporcionan diferentes características, así como también diferentes contaminantes (7).

Basados en el fuerte impacto de las características topográficas del implante de titanio en la oseointegración y en consideración de sus variaciones químicas, el objetivo de esta investigación fue evaluar la superficie rugosa de cinco sistemas de implantes de titanio comercialmente puro y establecer sus características químicas tanto de cuerpo como de superficie.

\section{MATERIAL Y MÉTODOS}

\section{MUESTRA Y DISEÑO DEL ESTUDIO}

Se realizo un estudio de tipo descriptivo doble ciego para la exploración de una muestra compuesta de quince implantes de titanio comercialmente puro con 3,75 $\mathrm{mm}$ de diámetro por $10 \mathrm{~mm}$ de largo. Los implantes utilizados fueron 1) Implamed ${ }^{\circledR}$ (Sterngold-Implamed dental Implant System Ltda., Attleboro, MA, USA), 2) SImplant ${ }^{\circledR}$ (S Serson Implant - S Serson Internacional,
São Paulo,SP, Brasil), 3) Master Screw ${ }^{\circledR}$ (Conexão - Sistema de Prótesis, São Paulo, SP Brasil), 4) INP ${ }^{\circledR}$ (INP Sistema Nacional de Implantes y Protesis, São Paulo, SP, Brasil) y 5) Titanium Fix ${ }^{\circledR}$ (AS Technology, São Paulo, $\mathrm{SP}$, Brasil). Los mismos se dividieron en cinco grupos, con tres unidades de muestra cada uno. Cada grupo fue denominado con letras del alfabeto, codificándose como grupos A, B, C, D y E, cada uno con subdivisiones numéricas de 1,2 y 3 . Las muestras fueron estudiadas en el "Centro de Caracterização e Desenvolvimento de Materiais (CCDM). UFSCar/UNESP"; de este modo, el investigador responsable por los análisis no fue capaz de identificar la empresa fabricante. Las técnicas utilizadas en esta investigación fueron basadas en Metalografía, Microscopia Electrónica de Barrido y Sistema de Energía Dispersa (MEV-EDS) acoplada a microanálisis y analizador de imágenes.

\section{MÉTODOS DE ANÁLISIS}

Inicialmente cada implante se removió de su envoltura, utilizando pinzas plásticas y guantes de látex, sin manipulación ni contaminación por agentes externos, trabajando con la siguiente metodología.

\section{Análisis para la determinación del tamaño de los granos}

Se utilizó un microscopio LEICA unido a un analizador de imágenes con software Quantimed 600 y la norma ASTM E 112-95 "Standar Test Methods for Determing Average Grain Size", con aumento de 200 veces, estableciendo escalas numéricas en micras para el análisis de cada uno de los campos. Luego del análisis de todos los capos necesarios para cubrir la totalidad de la superficie del implante se obtuvo un promedio que indicaba el tamaño de los granos.

\section{Análisis microestructural de la superficie}

Un implante de cada marca comercial fue seleccionado aleatoriamente y posicionado sobre un porta-muestras; posteriormente se fijo a este con adhesivo conductor a base de plata. A continuación, su superficie se analizó en un microscopio electrónico de barrido unido a un sistema de microanálisis (MEV-EDS), en la forma de electrones secundarios, con una aceleración de $15 \mathrm{kV}$, y una distancia media de 29 milímetros. Se analizaron áreas próximas al valle del paso de rosca (alma del implante) y al tope de las roscas de cada implante, en aumentos de 500 veces. Con este análisis 
se pudo observar la existencia de inclusiones metálicas o contaminantes (elementos en la superficie analizada que no correspondan a titanio u otro material señalado por el fabricante). La presencia de aluminio no se evaluó por ser inherente al proceso de preparación de las muestras. La existencia de inclusiones se estudio mediante un gráfico obtenido desde el analizador de imágenes que señalaba cuantitativamente la presencia del elemento. Para nuestra investigación, solo exponemos la presencia o ausencia de ellos.

\section{Análisis microestructural en corte transversal}

Un implante de cada marca comercial se cortó transversalmente por un "cut-off" (cortadora eléctrica), sobre refrigeración y con disco diamantado, insertados en resina de curado frío y lijados en máquina automática en secuencia de lijas (240, 320, 400 y 600 mesh), pulidos automáticamente con alúmina en solución a 0,1 e 0,05 $\mu \mathrm{m}$ y finalmente atacado químicamente con una solución de 3 $\mathrm{ml}$ de $\mathrm{HCL}$ (concentrado), $5 \mathrm{ml}$ de $\mathrm{HNO}_{3}$ (concentrado), 2 $\mathrm{ml}$ de $\mathrm{HF}(40 \%)$ y $190 \mathrm{ml}$ de $\mathrm{H}_{2} \mathrm{O}$ destilada, para revelar los granos de la microestructura. Después de estos procedimientos, fue realizado un baño superficial con oro para mejorar el contacto con el analizador de imágenes.

\section{RESULTADOS}

\section{Análisis para determinación del tamaño de los granos}

Se obtuvo diferentes variaciones de granos entre las muestras seleccionadas (Tabla 1). La figura 1 corres- ponde a las imágenes obtenidas del análisis microscópico de los tamaños de los granos, donde es posible observar las variaciones de tamaño entre los implantes estudiados. Objetivamente, del tamaño existente entre los implantes destaco Implamed ${ }^{\circledR}$ como el de menor tamaño de granos con $10,71 \mu \mathrm{m}$ y S-Implant ${ }^{\circledR}$ como el de granos superficiales de mayor tamaño con 20,24 $\mu \mathrm{m}$, duplicando el tamaño de Implamed $^{\circledR}$.

\section{Análisis metalográfica de la superficie}

Se realizó una búsqueda metalográfica idéntica de inclusiones en cada uno de los campos de todas las superficies analizadas. Se obtuvieron microfotografías de la superficie de las muestras que fueron analizadas en una visión panorámica, específicamente en el valle del paso de roscas y el tope de las roscas. De un modo general, todas las muestras presentaron picos elevados de titanio junto a picos de carbono y oxígeno.

$\mathrm{Al}$ análisis de los valles, encontramos carbono, oxígeno y silicio presentes en todas las muestras; al análisis de las inclusiones observadas en determinadas muestras, verificamos la presencia de sodio en Implamed $^{\circledR}$, carbono, oxígeno e silicio en Titanium Fix ${ }^{\circledR}$ AST e INP ${ }^{\circledR}$ y carbono, oxígeno, sodio y cloro en S-Implant ${ }^{\circledR}$ (Tabla 2).

$\mathrm{Al}$ análisis de los topes de rosca, encontramos carbono y oxígeno presente en todos los implantes, mientras que solo en Titanium $\mathrm{Fix}^{\circledR}$ AST fue encontrado silicio. Observamos también en Master Screw ${ }^{\circledR}$ la presencia de carbono, oxígeno, sodio, calcio y un alto pico de azufre; en la muestra de INP ${ }^{\circledR}$ también presento carbono, oxígeno e calcio.

TABLA 1.- TAMAÑ̃O DE LOS GRANOS DE SUPERFICIE DE CINCO MARCAS DE IMPLANTES DE TITANIO COIMERCIALMENTE PURO. SE ESTABLECE LA PRESENCIA DE GRANOS PEQUEÑO (IMPLAMED ${ }^{\circledR}$ Y MASTER SCREW ${ }^{\circledR)}$ MEDIO (TITANIUM FIX ${ }^{\circledR}$ AST) Y GRANDE (INP ${ }^{\circledR}$ Y S-IMPLANT ${ }^{\circledR}$ ).

\begin{tabular}{|l|c|c|c|c|c|}
\hline Grupo & Implamed $^{\circledR}$ & Master Screw $^{\circledast}$ & Titanium Fix $^{\circledR}$ AST & INP $^{\circledR}$ & S-Implant $^{\circledast}$ \\
\hline Tamaño granos (ìm) & 10,71 & 12,38 & 16,34 & 20,64 & 20,54 \\
\hline
\end{tabular}



Fig. 1: Imagen de la morfología de superficie obtenida para le evaluación del tamaño de granos utilizando MEV-EDS en aumento de 200 veces. A) Implamed ${ }^{\circledR}$; B) Master Screw ${ }^{\circledR}$; C) Titanium Fix ${ }^{\circledR}$; D) INP®; E) S-Implant ${ }^{\circledR}$. 


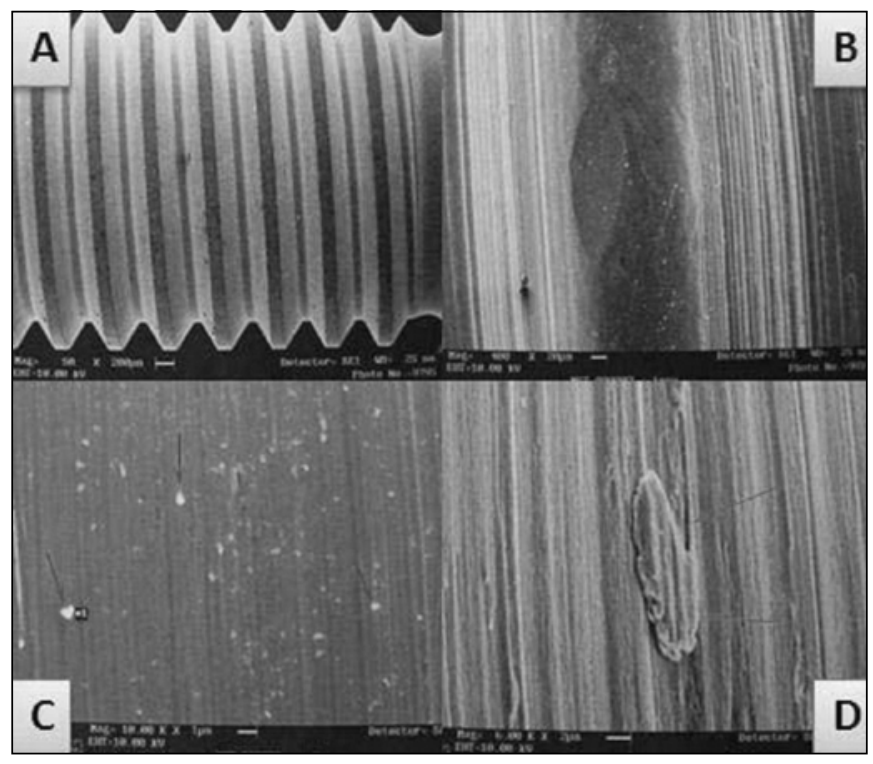

Fig.2: Imágenes utilizadas de MEV-EDS en el análisis de superficie para obtener información sobre inclusiones y microarquitectura. A) Implamed ${ }^{\circledR}$ : micrografía general de superficie (200 X, escala a 200 ìm). B) Master Screw ${ }^{\circledR}$ : micrografía de tope de rosca, presentando ranuras inherentes al proceso de fabricación ( $500 \mathrm{X}$, escala a 20 ìm). C) Titanium Fix ${ }^{\circledR}$ AST: micrografía de valle de rosca presentando inclusiones señaladas por las flechas de color azul; cada una de las zonas blancas representa una inclusión (5.000 X, escala a 1 ìm). D) INP ${ }^{\circledR}$ : micrografía de valle de rosca presentando un defecto en el proceso de fabricación señaladas por las flechas de color rojo (500 $X$, escala de 2 ìm).

\section{Análisis metalográfica en corte transversal}

Todas las muestras presentaron picos de carbono en su contenido. Implamed ${ }^{\circledR}$ presentaba inclusiones que contenían carbono, oxígeno, fierro, sodio, magnesio, silicio, potasio y calcio. Master Screw ${ }^{\circledR}$ presento inclusiones de carbono y fierro, mientras que INP $^{\circledR}$ contenían solamente carbono (Tabla 2).

\section{DISCUSIÓN}

Actualmente existe una gran cantidad de implantes y tratamientos de superficies al alcance de cualquier clínico. El titanio comercialmente puro y aleaciones de Ti-6Al-4V han sido ampliamente utilizados en implantología oral, y su empleo, en ausencia de tratamientos de superficie, aún es utilizado. Nuestra investigación se interesó en abordar estos implantes ya que existe mayor conocimiento y consenso de las etapas utilizadas en su elaboración, siendo más estandarizados los procesos de fabricación. Los tratamientos de superfi- cie varían en cuanto a las técnicas aplicadas por cada fabricante, haciéndolas poco comparables cuando se trata de analizar contaminantes de fabrica (8). Nuestro estudio utilizó MEV-EDS como herramienta principal para los análisis; este es uno más de los métodos para evaluar superficies de implantes; por otra parte, también existen cerca de 150 parámetros para calcular y caracterizar la microtopografía de superficie (9). En relación a la forma de analizar la superficie del implante, de un modo general Wennerberg \& Albrektsson (9) recomendaron la evaluación en las zonas de valles, topes y flancos de rosca, que son precisamente las que esta investigación reporta.

La interacción biológica entre la superficie de implantes y los componentes celulares son parcialmente conocidos. Si consideramos que estructuras celulares oscilan entre 1 y $100 \mu \mathrm{m}$, mientras que proteínas están en el rango de 0,001 a 0,01 $\mu \mathrm{m}$, el valor de superficies rugosas de menor tamaño en oseointegración es considerable (10). De hecho, superficies lisas son asociadas a un mayor crecimiento de epitelio y consecuentemente a un aumento de bolsas periimplantarias (11). Investigaciones han demostrado que superficies menores de $10 \mu \mathrm{m}$, han sido asociadas a mayor éxito clínico (12) y que el torque de remoción en superficies rugosas es mayor (13), demostrando cuantitativamente mayor contacto óseo; basado en esto, solo Implamed ${ }^{\circledR}$ y Master Screw ${ }^{\circledR}(10$ y $12 \mu \mathrm{m}$ aproximadamente) podrían llegar a tener una mayor influencia biológica en la interacción hueso-implante.

En el proceso de oseointegración existe una reacción electroquímica en la interfase implante-hueso que implica la incorporación de iones en la superficie del implante y la liberación de iones desde la misma superficie (14), llegando a observar titanio en regiones óseas que circunda el implante (15); si esta reacción ocurre con el titanio, es posible que también ocurra con materiales contaminantes como los analizados en este estudio, donde la liberación de tales iones metálicos podrían causar reacciones tóxicas o de hipersensibilidad en el corto o largo plazo (16). Por otra parte, Taborelli et al. (17) confirmaron la importancia de la camada de oxido de titanio para la incorporación de los implantes y para el íntimo contacto de su superficie con la del hueso; no sabemos la influencia que estos contaminantes podrían tener sobre esta camada. En nuestro estudio Implamed ${ }^{\circledR}$, Master Screw $^{\circledR}$ e INP ${ }^{\circledR}$ presentaron contaminaciones en el corte transversal, lo que puede indicar algún grado de contaminación durante la fabricación del implante o la manipulación del titanio. En relación al área existió 


\section{TABLA 2.- INCLUSIONES DE ELEMMNTOS EN DIFERENTES ZONAS ANALIZADAS DE LAS CINCO MARCAS DE IMPLANTES DE TITANIO COIMERCIALMENTE PURO. SE PUEDE OBSERVAR QUE EXISTE CONTAMINACIÓN EN TODOS LOS NIVELES DE INP $^{\circledR}$, MIENTRAS QUE IMPLAMED ${ }^{\circledR}$ PRESENTO LA MAYOR CANTIDAD DE CONTAIMINANTES}

\begin{tabular}{|c|c|c|c|c|c|c|c|c|c|c|c|c|c|c|c|}
\hline \multirow[t]{2}{*}{ Elementos } & \multicolumn{3}{|c|}{ Implamed $^{\circledR}$} & \multicolumn{3}{|c|}{ Master Screw } & \multicolumn{3}{|c|}{$\begin{array}{l}\text { Titanium } \\
\text { Fix }^{\circledR} \text { AST }\end{array}$} & \multicolumn{3}{|c|}{ INP $^{\circledR}$} & \multicolumn{3}{|c|}{ S-Implant ${ }^{\circledR}$} \\
\hline & $\mathbf{v}$ & $\mathbf{T}$ & $\mathbf{T r}$ & $\mathbf{v}$ & $\mathbf{T}$ & $\mathbf{T r}$ & $\mathbf{v}$ & $\mathbf{T}$ & $\mathbf{T r}$ & $\mathbf{v}$ & $\mathbf{T}$ & $\mathbf{T r}$ & $\mathbf{v}$ & $\mathbf{T}$ & $\mathbf{T r}$ \\
\hline Silicio & & & + & & & & + & & & + & & & & & \\
\hline Oxigeno & & & + & & + & & + & & & + & + & & + & & \\
\hline Sodio & + & & + & & + & & & & & & & & + & & \\
\hline Carbono & & & + & & + & & + & & & + & + & + & + & & \\
\hline Cloro & & & & & & & & & & & & & + & & \\
\hline Fierro & & & + & & & + & & & & & & & & & \\
\hline Magnesio & & & + & & & & & & & & & & & & \\
\hline Potasio & & & + & & & & & & & & & & & & \\
\hline Calcio & & & + & & + & & & & & & + & & & & \\
\hline Azufre & & & & & + & & & & & & & & & & \\
\hline $\begin{array}{l}\text { Total elementos } \\
\text { en inclusiones }\end{array}$ & 1 & - & 8 & - & 5 & 1 & 3 & - & - & 3 & 3 & 1 & 4 & - & - \\
\hline
\end{tabular}

mayor cantidad de inclusiones en la región de los valles de roscas ( 4 de los 5 implantes analizados) que en la región de los topes de rosca (2 de los 5 implantes analizados), lo que podría responder al hecho de que el proceso de limpieza final del implante, previo al proceso de esterilizado y embalaje, es insuficiente en el áreas de los valles. Los implantes con menos carga de impurezas fueron Titanium Fix ${ }^{\circledR}$ AST y S-Implant ${ }^{\circledR}$. Un hallazgo particular en este estudio es el hecho de que el implante con tamaño de partícula menor fue el que presento mayor cantidad de contaminantes; podría existir alguna relación entre estas dos situaciones, lo que deberemos estudiar con nuevas investigaciones.

En condiciones normales es frecuente encontrar contaminación con elementos orgánicos y no orgánicos en superficies de implantes (7), donde estos últimos estarían en función de la manufactura, limpieza, trata- miento de superficie, embalaje y esterilización (18). Aunque implantes con superficies de titanio comercialmente puro con diferentes tratamientos físicos de superficies no demostraron diferencia en términos de contacto hueso-implante (19), la presencia contaminantes metálicos si podría influenciarla. Por otra parte, se ha observado que la energía libre de superficie puede tener influencias en la capacidad de una mayor adhesión en la interface implante-hueso (20); las inclusiones de carbono, por ser hidrófobo, podrían disminuir la energía libre de la superficie del material impidiendo la adhesión de biomoléculas, células epiteliales, fibroblastos y osteoblastos (21). Las otras inclusiones pueden funcionar como células electrolíticas provocando cambios iónicos y corrosión en el titanio, interfiriendo en el proceso de unión química de la camada de biomoléculas (22). Estudios de seguimiento han demostrado oseointegración de implantes de superficie de titanio comercialmente puro (23), 
pero el efecto de los contaminantes en el largo plazo no ha sido estudiado. De esta forma podrían presentar desde ninguna complicación hasta alteraciones significativas de las propiedades mecánicas del implantes, sobre el comportamiento con los fenómenos de corrosión y finalmente sobre las características biológicas del sujeto.

\section{CONCLUSIÓN}

Las muestras de implantes analizadas fueron aceptables en su microarquitectura. Si bien estimamos que los implantes presentaran pequeñas variaciones en cuanto a la probable relación hueso-implante, no serían variaciones significativas al punto de interferir en la oseointegración de estos implantes. En relación a los contaminantes Implamed ${ }^{\circledR}$ presentó una gran cantidad de contaminantes, cuyas influencias en el largo plazo no están determinadas.

\section{BIBLIOGRAFÍA}

1. Han CH, Johansson CB, Wennerberg A., Albrektsson T. Quantitative and qualitative investigations of surface enlarged titanium and titanium alloy implants. Clin Oral Implants Res. 1998 Feb;9(1):1-10.

2. Masaki C, Schneider GB, Zaharias D, Stanford C. Effects of implant surface microtopography on osteoblast gene expression. Clin Oral Implants Res. 2005 Dec;16(6):6506

3. SulYT, Johansson CB, Röser K, Albrektsson T. Qualitative and quantitative observations of bone tissue reactions to anodized implants. Biomaterials. 2002 Apr;23(8):1809-17.

4. Grössner-Schreiber B, Griepentrong M, Haustein I, Müller WD, Lange KP, Briedigkeit H, Göbel Ub. Plaque formation on surface modified dental implants. An in vitro study. Clin Oral Implants Res. 2001 Dec;12(6):54351.

5. Ameen AP, Short RD, Johns R, Schwach G. The surface analysis of implant materials. 1 .The surface composition of a titanium dental implant material. Clin Oral Implants Res. 1993 Sep; 4(3):144-50.

6. Edwards BN, Gold BR. Analysis of surface cleanliness of three commercial dental implants. Biomaterials. 1992;13(11):775-80.
7. Placko HE, Mishra S, Weimer JJ, Lucas LC. Surface characterization of titanium-based implant materials. Int J Maxillofac Implants. 2000 May-Jun; 15(3):355-63.

8. Bächle M, Kohal RJ. A systematic review of the influence of different titanium surfaces on proliferation, differentiation and protein synthesis of osteoblast-like MG63 cells. Clin Oral Implants Res. 2004 Dec;15(6):683-92.

9. Wennerberg A, Albrektsson T. Suggested guidelines for the topographic evaluation of implant surfaces. Int J Oral Maxillofac Implants. 2000 May-Jun;15(3):331-44.

10. Shalabi MM, Gortemaker A, Van't Hof MA, Jansen JA, Creugers NH. Implant surface roughness and bone healing: a systematic review. J Dent Res. 2006 Jun; 85(6):496-500.

11. Bollen CM, Papaioanno W, Van Eldere J, Schepers E, Quirynen M, van Steenberghe D. The influence of abutment surface roughness on plaque accumulation and peri-implant mucositis. Clin Oral Implants Res. 1996 Sep;7(3):201-11.

12. Glauser R, Ruhstaller P,Windisch S, Zembic A, Lundgren A, Gottlow J, Hämmerle CH. Immediate occlusal loading of Brånemark System TiUnite implants placed predominantly in soft bone: 4-year results of a prospective clinical study. Clin Implant Dent Relat Res. 2005;7 Suppl 1:S52-9.

13. Gotfredsen K, Berglundh T, Lindhe J. Anchorage of titanium implants with different surface characteristics: an experimental study in rabbits. Clin Implant Dent Relat Res. 2000;2(3):120-8.

14. Sundgren JE, Bodö P, Lundström I, Berggren A, Hellem S. Auger electron spectroscopic studies of stainlesssteel implants. J Biomed Mater Res. 1985 Jul-Aug;19 (6):663-71.

15. Wennerberg A, Ide-Ektessabi A, Hatkamata S, Sawase T, Johansson C, Albrektsson T, et al.Titanium release from implants prepared with different surface roughness. Clin Oral Implants Res. 2004 Oct; 15(5):505-12.

16. Merritt K, Brown SA. Distribution of cobalt chromium wear and corrosion products and biologic reactions. Clin Orthop Relat Res. 1996 Aug; (329 Suppl):S233-43.

17. Taborelli M, Jobin M, François $P$, Vaudaux P, Tnetti M, Szmukler-Moncler S, et al. Influence of surface treatments developed for oral implants on the physical 
and biological properties of titanium. (I) Surface characterization. Clin Oral Implants Res. 1997 Jun;8(3): 208-16.

18. Kilpadi DV, Lemons JE, Liu J, Raikar GN, Weimer JJ, Vohra Y. Cleaning and heat-treatment effects on unalloyed titanium implant surfaces. Int. J Oral Maxillofac Implants. 2000 Mar-Apr;15(2):219-300.

19. Kim YH, Koak JY, Chang IT, Wennerberg A, Heo SJ. A histomorphometric analysis of the effects of various surface treatment methods on osseointegration. Int J Oral Maxillofac Implants 2003 May-Jun; 18(3):349-56.

20. Hamamoto N, Hamamoto Y, Nakajima T, Ozawa H. Histological, histocytochemical and ultrastructural study on the effects of surface charge on bone formation in the rabbit mandible. Arch Oral Biol. 1995 Feb;40(2):97-106.

21. Keller JC, Linn JE. Characterization of sterilized CP titanium implant surfaces. Int J Oral Maxillofac Implants. 1990 Winter;5(4):360-7.
22. Olefjord I, Hansson S. Surface analyses of four dental implant system. Int J Oral Maxillofac Implants. 1993;8 (1):32-40.

23. Boioli LT, Penaud J, Miller N. A meta-analytic, quantitative assessment of osseointegration establishment and evolution of submerged and non-submerged endosseous titanium oral implants. Clin Oral Implants Res. 2001 Dec;12(6):579-88.

\section{CORRESPONDENCIA}

Prof. Dr. Jose Ricardo de Albergaria Barbosa

Av. Limeira 901, Caixa Postal 52

CEP 13414-903

Piracicaba

SP, Brasil

Tel.: (19) 2106-5200

Fax.: (19) 3421-0144

solate@fop.unicamp.br 